\title{
Impact of disease activity on health-related quality of life in systemic lupus erythematosus - a cross-sectional analysis of the Swiss Systemic Lupus Erythematosus Cohort Study (SSCS)
}

Benjamin Chaigne ${ }^{1}$, Carlo Chizzolini ${ }^{1}$, Thomas Perneger ${ }^{2}$, Marten Trendelenburg ${ }^{3}$, Uyen Huynh-Do ${ }^{4}$, Eric Dayer ${ }^{5}$, Thomas Stoll ${ }^{6}$, Johannes von Kempis ${ }^{7}$, Camillo Ribi ${ }^{8^{*}}$ (D), for the Swiss Systemic Lupus Erythematosus Cohort Study Group

\begin{abstract}
Background: To assess the impact of disease activity on health-related quality of life (HRQoL) in systemic lupus erythematosus (SLE).

Methods: Cross-sectional study of patients included in the Swiss SLE Cohort Study between April 2007 and June 2014. HRQoL outcomes were based on the Medical Outcome Study Short Form 36 (SF-36). Disease activity was assessed by the SLE Disease Activity Index score with the Safety of Estrogens in SLE National Assessment modification (SELENA-SLEDAI) and by the physican's global assessment (PGA).

Results: Of the 252 patients included, 207 (82\%) were women. Median [interquartile range (IQR)] age was 43 [32-57] years. SLE was active in 125 patients (49.6\%). Median [IQR] mental component summary (MCS) in active vs inactive SLE was 40.0 [30.2-51.0] compared to 47.3 [39.2-52.8] $(p<0.01)$ and median [IQR] physical component summary (PCS) 43.7 [37.0-52.8] compared to 49.1 [38.4-55.6], respectively $(p<0.05)$. Increase in SELENA-SLEDAI or increase in PGA were negatively correlated with PCS and/or MCS. After adjusting for gender, age and disease duration, disease activity impacted on both PCS and MCS and all subscales except general health. Active lupus nephritis and musculoskeletal involvement were associated with physical limitations and emotional problems, increased bodily pain and poor social functioning. Low complement and/or presence of anti-dsDNA antibodies were associated with increased fatigue and reduced mental health.
\end{abstract}

Conclusions: In patients with SLE, HRQoL is reduced in those with active disease. Impact of disease activity on HRQoL dimensions depends on SELENA-SLEDAI system components.

Keywords: Systemic Lupus Erythematosus, Disease activity, Damage, Health-related quality of life

\footnotetext{
* Correspondence: camillo.ribi@chuv.ch

${ }^{8}$ Clinical Immunology and Allergy, University Hospital Lausanne, Rue du

Bugnon 46, Lausanne 1011, Switzerland

Full list of author information is available at the end of the article
} 


\section{Background}

Systemic lupus erythematosus (SLE) is a chronic autoimmune disease that mainly affects women. This multifactorial disease arises in genetically susceptible individuals upon activation of the innate and adaptive immune system through internal and environmental factors [1]. Clinical presentation is diverse and may include joint, skin, kidney, neurological or hematological involvement [2]. Sustained inflammation in tissues may lead to organ dysfunction and failure. Fatigue and pain are also prominent complaints in SLE patients [3, 4]. SLE activity and damage in addition to fatigue and pain impact on patients quality of life (QoL) [5, 6]. Thus, treatment in SLE should not only aim at decreasing disease activity and damage accrual but also at improving health-related quality of life (HRQOL) [7].

Both lupus-specific QoL questionnaires and the generic Medical Outcomes Study 36-Item Short Form Health Survey (SF-36) have been used to assess HRQOL in SLE [8]. Most SLE studies have used the SF-36, showing that this tool reliably assess HRQOL in this disease [5, 9-11]. SF-36 outcomes have been used as endpoints for treatment efficacy or as prognostic marker $[7,10,12]$. The influence of disease activity on HRQOL is still debated, possibly because only a few studies have examined disease-related organ involvement in relationship with HRQOL [13-19]. Herein we aimed at assessing baseline HRQOL in a cohort of adult SLE patients $[2,20]$ and to correlate SF36 scores with global disease activity. In order to further emphasize the possible influence of disease activity on SLE, we assessed the impact of organ involvement on HRQOL.

\section{Methods}

\section{Patients}

Cross-sectional data were collected on patients sequentially included in the Swiss SLE Cohort Study (SSCS) between April 2007 and June 2014. Inclusion criteria were: age $\geq 18$ years, diagnosis SLE according to the updated ACR classification criteria [21, 22] or the SLICC 2012 criteria [23], completed SF-36 and corresponding data on disease activity, manifestations and treatment. The cohort study was approved by the ethics review boards of all participating institutions and all patients gave written informed consent. Patients included originated from Clinical Immunology, Internal Medicine, Nephrology, and Rheumatology tertiary care centers located both in the French and German-speaking regions of Switzerland.

\section{Data collection}

Data on patient's age, sex, ethnicity and family history of SLE, dates of first lupus manifestation and diagnosis, clinical and biological characteristics at baseline, disease activity, laboratory parameters, treatment modalities and co-morbidity were collected. HRQoL was assessed by the SF-36 Version $1[24,25]$. This tool comprises 8 dimension-scales: physical function (PF), role limitations due to physical problems (role physical, RP), bodily pain $(\mathrm{BP})$, general health $(\mathrm{GH})$, vitality $(\mathrm{VT})$, social function (SF), role limitations due to emotional problems (role emotional, RE), and mental health $(\mathrm{MH})$. Each scale ranges from 0 (lowest possible score) to 100 (highest possible score). These 8 dimensions can be summarized into two global scores, the physical component summary (PCS) and the mental component summary (MCS). Expected SF-36 outcomes for an age- and sex-matched population were generated using an algorithm based on the results of a survey conducted on 1200 adults in Western Switzerland [26]. Disease activity was assessed independently of the SF-36 on the same day, using the Systemic Lupus Erythematosus Disease Activity Index (SLEDAI) score with the Safety of Estrogens in Lupus Erythematosus National Assessment (SELENA) modification [27]. Patients were classified in 2 groups according to their SELENA-SLEDAI: inactive SLE (SLEDAI < 4) and active SLE (SLEDAI $\geq 4)$. Disease activity was also assessed by the Physician's Global Assessment (PGA) score with a 4-point-Likert-scale, ranging from 0 (inactive disease) to 3 (very active disease). Medication was detailed for disease-modifying drugs (DMARD's) taken, which were classified in three groups: systemic glucocorticosteroids (GC), anti-malarials (AM) and immunosuppressive agents (IS). All parameters reflected the 4-week period preceding completion of the SF-36 [28].

\section{Primary outcomes}

Primary outcomes were the differences in the eight SF36 dimension scales at baseline in patients with active and inactive disease.

\section{Statistical analysis}

Quantitative variables were expressed as the median \pm interquartile range (IQR) and non-parametric statistics were used to analyze the data. P-values $<0.05$ (two-sided) were considered significant. A linear regression model was used for multivariate analysis, with SF-36 dimension as dependent variables and age, sex, disease duration and SELENA-SLEDAI system components as independent variables. The purpose of this analysis was to identify which of the SELENA-SLEDAI system component were most strongly associated with differences in SF-36 dimensions. Statistical analysis was performed using GraphPad Prism version 6.00 (GraphPad Software, La Jolla, CA), and SPSS Version 22 (IBM Corp Armonk, NY). Spydergrams were generated using Excel Version 14 (Microsoft, Redmond, Washington). 


\section{Results}

Two hundred and fifty-two patients met the inclusion criteria. Patients' baseline characteristics are shown in Table 1. Approximately half of the patients had inactive disease (Table 1). Those with active disease defined as SELENA-SLEDAI $\geq 4$ accordingly had a higher PGA score. They were more often smokers and positive for anti-Sm antibodies. They also had higher ESR values, lower hemoglobin and serum albumin levels and received more often GC and higher average daily prednisone doses (Table 1). HRQoL outcomes in SLE were significantly reduced compared to what is expected in the age- and

Table 1 Characteristics of 252 patients with sytemic lupus erythematosus with inactive and active disease at inclusion

\begin{tabular}{|c|c|c|c|c|}
\hline Characteristics & All $(N=252)$ & Inactive $^{a}(N=127)$ & Active $^{a}(N=125)$ & $p$-value \\
\hline Sex, women/men (\%) & $207 / 45(82 / 18)$ & $104 / 23(82 / 18)$ & $103 / 22(82 / 18)$ & 1.00 \\
\hline Age, median [IQR], years & $43[32-57]$ & $45[32-59]$ & $42[32-55]$ & 0.23 \\
\hline Body mass index, median [IQR], kg/m2 & $24.1[21.2-27.4]$ & $24.4[22.0-28.5]$ & $24.0[20.9-26.7]$ & 0.07 \\
\hline Smoking, no (\%) & $46(18)$ & $15(12)$ & $31(25)$ & $<0.01$ \\
\hline Disease duration, median [IQR], years & $6.2[2.6-14.3]$ & $6.0[2.9-12.8]$ & $6.6[2.3-15.1]$ & 0.45 \\
\hline \multicolumn{5}{|l|}{ ACR criteria } \\
\hline Malar rash, no (\%) & $92(37)$ & $42(33)$ & $50(40)$ & 0.30 \\
\hline Discoid rash, no (\%) & $46(18)$ & $28(22)$ & $18(14)$ & 0.14 \\
\hline Photosensitivity, no (\%) & $121(48)$ & $58(46)$ & $63(50)$ & 0.53 \\
\hline Nasopharyngeal ulcers, no (\%) & $70(28)$ & $35(28)$ & $35(28)$ & 1.00 \\
\hline Arthritis, no (\%) & $173(69)$ & $83(65)$ & $90(72)$ & 0.28 \\
\hline Pleuritis, no (\%) & $58(23)$ & $26(21)$ & $32(26)$ & 0.45 \\
\hline Pericarditis, no (\%) & $44(18)$ & $23(18)$ & $21(17)$ & 0.87 \\
\hline Renal disorder, no (\%) & $90(36)$ & $39(31)$ & $51(41)$ & 0.12 \\
\hline Seizures, no (\%) & $12(5)$ & $4(3)$ & $8(6)$ & 0.25 \\
\hline Psychosis, no (\%) & $13(5)$ & $6(5)$ & $7(6)$ & 0.78 \\
\hline Hematologic disorder, no (\%) & $155(62)$ & $73(58)$ & $82(66)$ & 0.20 \\
\hline Antinuclear antibodies positive, no (\%) & $246(98)$ & $125(98)$ & $121(97)$ & 0.44 \\
\hline Anti-Sm antibody positive, no (\%) & $38(15)$ & $13(10)$ & $25(20)$ & 0.035 \\
\hline Anti-dsDNA antibodies positive, no (\%) & $150(60)$ & $68(54)$ & $82(66)$ & 0.055 \\
\hline Anti-phospholipid antibodies positive, no (\%) & $108(43)$ & $55(43)$ & $53(42)$ & 0.90 \\
\hline \multicolumn{5}{|l|}{ Laboratory values } \\
\hline Haemoglobin, median [IQR], g/L & 130 [117-137] & $131[122-138]$ & 128 [113-135] & 0.011 \\
\hline Leukocytes, median [IQR], G/L & $5.9[4.5-7.7]$ & $5.6[4.3-7.5]$ & $6.2[4.6-8.35]$ & 0.12 \\
\hline Platelets, median [IQR], G/L & 238 [189-294] & 240 [189-285] & 236 [185-303] & 0.97 \\
\hline Plasma creatinin, median $[\mathrm{IQR}], \mu \mathrm{mol} / \mathrm{L}$ & $70[61-87]$ & $70[61-86]$ & 70 [61-92] & 0.64 \\
\hline Serum albumin, median $[\mathrm{IQR}], \mathrm{g} / \mathrm{L}$ & $39.2[36.6-42.0]$ & $40.1[38.8-42.7]$ & 38 [35.0-40.3] & $<0.0001$ \\
\hline Erythrocyte sedimentation rate, median [IQR], $\mathrm{mm} / 1^{\text {st }}$ hour & $12[6-29]$ & $10[5-26]$ & $14[7-34]$ & 0.013 \\
\hline \multicolumn{5}{|l|}{ Disease activity and damage } \\
\hline Physician global assesment, median [IQR] & $0[0-1]$ & $0[0-1]$ & $1[0-1.25]$ & $<0.0001$ \\
\hline SELENA-SLEDAI, median [IQR] & $3[0.25-8]$ & $1[0-2]$ & $8[4-12]$ & $<0.0001$ \\
\hline \multicolumn{5}{|l|}{ Treatment } \\
\hline Oral glucocorticosteroids, no (\%) & $141(56.0)$ & $55(43.3)$ & $85(68)$ & $<0.0001$ \\
\hline Daily prednisone equivalent, median [IQR], mg & $7.5[5-12]$ & $5[5-7.5]$ & $9[5-20]$ & $<0.0001$ \\
\hline Antimalarials, no (\%) & $178(71)$ & $91(71)$ & $87(70)$ & 0.68 \\
\hline Immunosuppressants, no (\%) & $120(48)$ & $55(43)$ & $65(52)$ & 0.21 \\
\hline
\end{tabular}

SD Standard deviation, IQR Interquartile range, NS non significant, SELENA-SLEDAI Systemic Lupus Erythematosus Disease Activity Index (SLEDAI) score with the Safety of Estrogens in Lupus Erythematosus National Assessment (SELENA), SDI Systemic Lupus International Collaborating Clinics/American College of Rheumatology Damage Index

anactive disease at baseline was defined by a SELENA-SLEDAI $<4$ and active disease by a SELENA-SLEDAI $\geq 4$ 
sex-matched Swiss general population: Median [IQR] PCS in SLE was 46.0 [37.9-54.4] in contrast to expected 52.0 [46.8-57.2], and median [IQR] MCS in SLE was 44.5 [33.6-52.1], in contrast to expected 50.3 [48.7-51.9] (both $p<0.001)$. Except for RE, all other SF-36 dimensions were significantly lower in SLE compared to the expected results in the general population (Fig. 1). Within the SLE group and among general characteristics, only age and body mass index appeared to be negatively correlated with HRQoL outcomes, whereas gender, disease duration and smoking status were not (Table 2). Advanced age negatively impacted mainly on PF and BP, with a median scale of 78.6 and 66.4 in individuals younger than 35 years and 62.3 and 54.5 in those older than 55 years $(p<0.001$ and $p=0.002$ ), respectively. Although to a lesser extent than age, an increase in body mass index also negatively correlated with HRQoL, in particular with PF and BP (Table 2).

Disease activity had a negative influence on all dimensions of HRQOL, except on GH perception (Fig. 2; Table 2). Accordingly, patients with active SLE had significantly lower MCS (40.0 [30.2-51.0]) and lower PCS (43.7 [37.0-52.8]), compared to patients with inactive disease (MCS 47.3 [39.2-52.8], $p<0.01$ ), and PCS 49.1 [38.4-55.6], $p<0.05)$. The association between active SLE and poor HRQoL was confirmed when relying on PGA for disease activity (Fig. 2; Table 2). However, the correlation between BP and disease activity was stronger when assessed by PGA, compared to SELENA-SLEDAI. One the other hand, SELENA-SLEDAI correlated more closely with the RE dimension than PGA. SELENASLEDAI correlated more strongly with MCS and PGA with PCS (Table 2).

Among biological activity parameters, an elevated erythrocyte sedimentation rate negatively correlated with most SF-36 dimensions, while hemoglobin and serum albumin levels correlated positively with some (Table 2).

Regarding SLE treatment, GC and IS were associated with active disease, while AM were not (Table 1). Use of GC at the time of visit was associated with a decrease in particular in the RP dimension, with a mean score of 43.8 compared to 66.4 in patients not taking GC $(p<0.001)$.

After adjusting for gender, age and disease duration, disease activity measured by SELENA-SLEDAI impacted on both summary component scales of the SF-36 and all dimension, except GH (Table 3). The effects of the nine SELENA-SLEDAI organ systems on HRQoL are shown in Table 4. (and detailed in Additional file 1: Table S1). Activity in the musculoskeletal, renal and immunological components significantly affected one or more SF-36 dimensions, while there was no significant impact of constitutional signs, serositis, active cutaneous, vascular, and central nervous or hematologic involvement. Musculoskeletal SLE activity defined as arthritis or myositis was strongly associated with BP, but also negatively affected RP, RE and SF, as well as MH. Renal SLE activity defined by hematuria, pyuria, new or increasing proteinuria and/or presence of urinary casts was negatively associated with RE and $\mathrm{RP}$ subscales, and to a lesser extent with BP, VT and SF. Immunologic activity defined by low complement levels and/or presence of anti-dsDNA antibodies had a negative impact on VT and MH. Interestingly, the influence of musculoskeletal and renal activity on HRQoL did not depend on age, whereas the impact of vascular involvement became non-significant and that of immunologic activity substantial once adjusted for age, sex and disease duration.

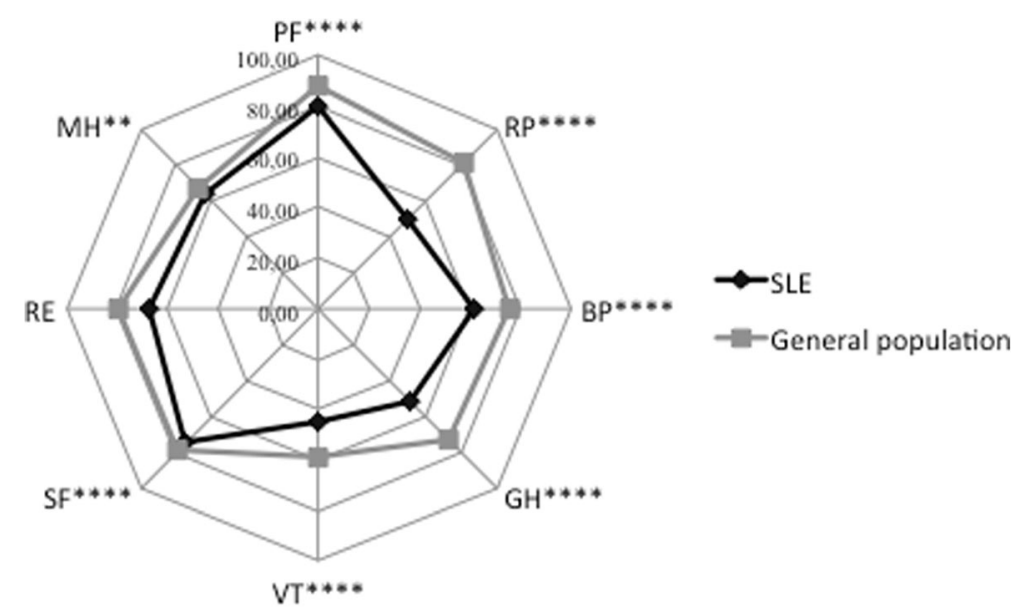

Fig. 1 Spydergrams of the eight baseline SF-36 dimensions in 252 patients included in the Swiss Systemic Lupus Erythematosus Cohort Study compared to the expected values in an age- and sex-matched sample of the Swiss general population. PF physical function, RP role physical, BP bodily pain, GH general health, VT vitality, SF social function, RE role emotional, MH mental health. ${ }^{*} p<0.05,{ }^{* *} p<0.01,{ }^{* * *} p<0.001,{ }^{* * * *} p<0.0001$ 
Table 2 Spearman's correlations between baseline characteristics and the eight dimensions and component summaries of the SF-36 in 252 patients with systemic lupus erythematosus

\begin{tabular}{lllllllllll}
\hline Disease characteristics & PF & RP & BP & GH & VT & SF & RE & MH & PCS & MCS \\
\hline Age at assessment & $-0.27^{* * * *}$ & -0.08 & $-0.18^{* *}$ & -0.05 & -0.01 & 0.02 & -0.07 & $0.15^{*}$ & $-0.24^{* * *}$ & 0.06 \\
Body mass index & $-0.25^{* * *}$ & -0.12 & $-0,16^{*}$ & -0.07 & -0.11 & -0.13 & $-0.15^{*}$ & 0.01 & $-0.18^{* *}$ & -0.05 \\
Disease duration & $0.14^{*}$ & 0.13 & 0.02 & 0.03 & 0.11 & 0.06 & 0.03 & $0.14^{*}$ & 0.12 & 0.02 \\
SELENA-SLEDAI & -0.07 & $-0.27^{* *}$ & $-0.22^{* *}$ & -0.09 & $-0.15^{*}$ & $-0.14^{*}$ & $-0.27^{* *}$ & $-0.16^{*}$ & $-0.14^{*}$ & $-0.21^{* * *}$ \\
PGA & $-0.17^{* *}$ & $-0.23^{* * *}$ & $-0.32^{* * *}$ & -0.08 & $-0.13^{*}$ & $-0.13^{*}$ & $-0.19^{* *}$ & $-0.16^{*}$ & $-0.21^{* * *}$ & $-0.14^{*}$ \\
ESR & $-0.26^{* * *}$ & $-0.24^{* * *}$ & $-0.23^{* * *}$ & -0.11 & -0.13 & $-0.15^{*}$ & $-0.21^{* *}$ & $-0.14^{*}$ & $-0.23^{* * *}$ & $-0.14^{*}$ \\
Haemoglobin & $0.16^{*}$ & $0.19^{* *}$ & 0.07 & 0.04 & 0.05 & 0.09 & $0.14^{* *}$ & 0.01 & $0.14^{*}$ & 0.06 \\
Serum albumin level & 0.06 & $0.16^{*}$ & 0.12 & 0.01 & -0.01 & 0.14 & 0.11 & -0.04 & 0.12 & 0.02 \\
Daily prednisone equivalent & -0.03 & -0.16 & -0.17 & 0.09 & -0.01 & $-0.18^{*}$ & -0.10 & -0.09 & -0.08 & -0.10 \\
\hline
\end{tabular}

ESR Erythrocyte sedimentation rate, PGA Physician's global assessment, SELENA-SLEDAl Systemic Lupus Erythematosus Disease Activity Index (SLEDAI) score with the Safety of Estrogens in Lupus Erythematosus National Assessment (SELENA), SDI Systemic Lupus International Collaborating Clinics/American College of Rheumatology Damage Index, $P F$ physical function, $R P$ role physical, $B P$ bodily pain, GH general health, $V T$ vitality, $S F$ social function, $R E$ role emotional, $M H$ mental health, $P C S$ Physical Component Summary, MCS Mental Component Summary

${ }^{*} p<0.05,{ }^{* *} p<0.01,{ }^{* * *} p<0.001,{ }^{* * *} p<0.0001$
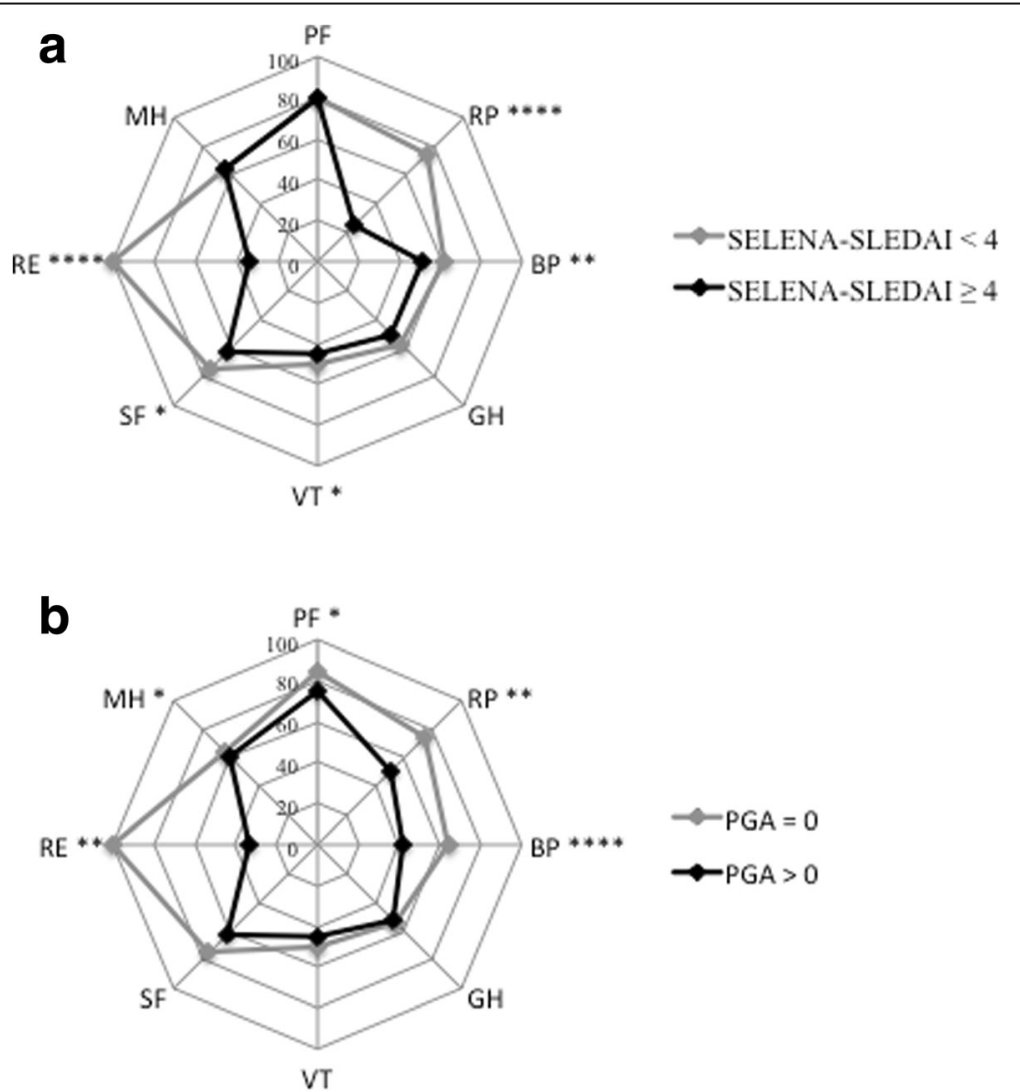

Fig. 2 Spydergrams of the eight baseline SF-36 dimensions in 252 patients included in the Swiss Systemic Lupus Erythematosus Cohort Study according to disease activity measures including SELENA-SLEDAI (a) and PGA (b) scores. SELENA-SLEDAI = Systemic Lupus Erythematosus Disease Activity Index (SLEDAI) score with the Safety of Estrogens in Lupus Erythematosus National Assessment (SELENA); PGA = Physican's global assessment; PF = physical function; $\mathrm{RP}=$ role physical; $\mathrm{BP}=$ bodily pain; $\mathrm{GH}=$ general health; $\mathrm{VT}=$ vitality; $\mathrm{SF}=$ social function; $\mathrm{RE}=$ role emotional; $\mathrm{MH}=$ mental health. ${ }^{*} p<0.05$, ${ }^{* *} p<0.01,{ }^{* * *} p<0.001$, ${ }^{* * *} p<0.001$ 
Table 3 Linear regression analysis on health-related quality of life outcomes in 252 patients with systemic lupus erythematosus in regard with disease activity age, sex and disease duration

\begin{tabular}{|c|c|c|c|c|c|c|}
\hline \multirow[t]{3}{*}{ Outcomes } & \multicolumn{3}{|l|}{ Predictors } & & & \\
\hline & \multicolumn{3}{|c|}{ SELENA-SLEDAl, unadjusted $(N=252)$} & \multicolumn{3}{|c|}{ SLEDAl, adjusted for age, sex and disease duration $(N=225)$} \\
\hline & $\begin{array}{l}\text { Difference in SF-36 score } \\
\text { for a 1-point increase in } \\
\text { SELENA-SLEDAl }\end{array}$ & 95\%-C.I. & $p$ & $\begin{array}{l}\text { Difference in SF-36 score } \\
\text { for a 1-point increase in } \\
\text { SELENA-SLEDAl }\end{array}$ & 95\%-C.I. & $p$ \\
\hline Physical function & -0.57 & $(-1.05,-0.10)$ & 0.019 & -0.66 & $(-1.12,-0.19)$ & 0.006 \\
\hline Role physical & -1.86 & $(-2.63,-1.09)$ & $<0.001$ & -1.85 & $(-2.63,-1.07)$ & $<0.001$ \\
\hline Bodily pain & -1.16 & $(-1.68,-0.64)$ & $<0.001$ & -1.27 & $(-1.79,-0.74)$ & $<0.001$ \\
\hline General health & -0.31 & $(-0.70,0.08)$ & 0.12 & -0.27 & $(-0.67,0.13)$ & 0.19 \\
\hline Vitality & -0.48 & $(-0.86,-0.10)$ & 0.014 & -0.46 & $(-0.86,-0.07)$ & 0.022 \\
\hline Social function & -0.72 & $(-1.19,-0.25)$ & $<0.001$ & -0.71 & $(-1.20,-0.22)$ & 0.005 \\
\hline Role emotional & -1.92 & $(-2.73,-1.11)$ & $<0.01$ & -1.86 & $(-2.70,-1.01)$ & $<0.001$ \\
\hline Mental health & -0.48 & $(-0.84,-0.13)$ & 0.008 & -0.46 & $(-0.81,-0.11)$ & 0.011 \\
\hline Mental component summary & -0.31 & $(-0.50,-0.12)$ & 0.002 & -0.34 & $(-0.53,-0.15)$ & $<0.001$ \\
\hline Physical component summary & -0.36 & $(-0.57,-0.15)$ & 0.001 & -0.33 & $(-0.55,-0.11)$ & 0.003 \\
\hline
\end{tabular}

SELENA-SLEDAI Systemic Lupus Erythematosus Disease Activity Index (SLEDAI) score with the Safety of Estrogens in Lupus Erythematosus National Assessment (SELENA)

\section{Discussion}

This study shows a reduction in most HRQoL outcomes in patients with active SLE, when assessed by the SF-36. Global disease activity is a strong predictor of HRQoL, even when adjusted for other factors such as age, sex and disease duration. While nearly all dimension of the SF-36 appear reduced in SLE in contrast to what is expected in an age- and gender-matched general population, with the SLE group active disease defined by a SELENA-SLEDAI $\geq 4$ has a dramatic effect on the dimensions that reflect the patient's role limitations. The relation between global SLE activity and HRQoL was assessed by other cross-sectional as well as longitudinal studies, with conflicting results [13-19]. For instance, two studies by Hanly and Gladman relying on the SF-20 $[13,14]$ did not find a correlation between disease activity and HRQOL. On the other hand, Stoll et al. using the SF-36 reported a significant association between disease activity assessed by the British Isles Lupus Activity Group System (BILAG) and HRQoL [15].

In this work, we aimed at further deciphering which dimensions were affected by global disease activity and SELENA-SLEDAI system components. Our results show that the SELENA-SLEDAI score with a 4-week window

Table 4 Impact of disease activity by organ systems assessed through the SELENA-SLEDAI on health-related quality of life outcomes in 252 patients with systemic lupus erythematosus

\begin{tabular}{|c|c|c|c|c|c|c|c|c|c|c|c|c|c|c|c|c|}
\hline \multirow{2}{*}{$\frac{\text { Predictors }}{\text { Unadjusted }(N=252)}$} & \multicolumn{16}{|c|}{ Outcomes } \\
\hline & PF & & $\mathrm{RP}$ & & $\mathrm{BP}$ & & $\mathrm{GH}$ & & VT & & SF & & RE & & $\mathrm{MH}$ & \\
\hline & B & $p$ & B & $p$ & B & $\mathrm{p}$ & B & $p$ & B & $p$ & B & $p$ & B & $P$ & B & $p$ \\
\hline Musculoskeletal & -6.75 & 0.11 & -18.45 & 0.008 & -21.89 & $<0.001$ & -4.84 & 0.15 & -4.01 & 0.23 & -8.80 & 0.032 & -20.27 & 0.005 & -6.85 & 0.027 \\
\hline Vascular & -27.12 & 0.019 & -39.45 & 0.040 & -12.96 & 0.32 & -7.51 & 0.43 & -7.13 & 0.44 & -4.79 & 0.68 & -32.58 & 0.11 & 1.47 & 0.86 \\
\hline Renal & -5.35 & 0.16 & -20.68 & 0.001 & -10.07 & 0.02 & -1.87 & 0.54 & -6.88 & 0.022 & -9.99 & 0.007 & -24.41 & $<0.001$ & -3.00 & 0.29 \\
\hline Immunologic & 1.88 & 0.56 & -4.96 & 0.36 & 2.24 & 0.54 & 0.04 & 0.99 & -4.67 & 0.070 & -2.08 & 0.51 & -8.06 & 0.15 & -4.14 & 0.084 \\
\hline Adjusted $^{\mathrm{a}}(\mathrm{N}=225)$ & PF & & $\mathrm{RP}$ & & $\mathrm{BP}$ & & $\mathrm{GH}$ & & VT & & SF & & RE & & $\mathrm{MH}$ & \\
\hline SLEDAI organ group & B & $P$ & B & $P$ & B & $\mathrm{p}$ & B & $P$ & B & $P$ & B & $P$ & B & $P$ & B & $P$ \\
\hline Musculoskeletal & -7.40 & 0.069 & -16.29 & 0.020 & -21.85 & $<0.001$ & -3.77 & 0.27 & -3.40 & 0.32 & -8.39 & 0.050 & -17.60 & 0.019 & -6.35 & 0.038 \\
\hline Vascular & -15.29 & 0.22 & -34.71 & 0.10 & -6.98 & 0.63 & -4.56 & 0.66 & -6.24 & 0.54 & -1.76 & 0.89 & -32.29 & 0.16 & -1.75 & 0.85 \\
\hline Renal & -6.41 & 0.09 & -21.99 & 0.001 & -11.57 & 0.01 & -1.52 & 0.63 & -7.26 & 0.022 & -9.89 & 0.012 & -25.47 & $<0.001$ & -2.43 & 0.40 \\
\hline Immunologic & -1.83 & 0.59 & -6.60 & 0.26 & -0.68 & 0.86 & -0.97 & 0.73 & -6.09 & 0.030 & -3.34 & 0.35 & -8.06 & 0.19 & -5.14 & 0.041 \\
\hline
\end{tabular}

$B$ Regression coefficient: Difference in the SF-36 subscale in patients with organ dysfunction, $P F$ physical function, $R P$ role physical, $B P$ bodily pain, $G H$ general health, VT vitality, SF social function, RE role emotional, MH mental health, SELENA-SLEDAI Systemic Lupus Erythematosus Disease Activity Index (SLEDAI) Score with the Safety of Estrogens in Lupus Erythematosus National Assessment (SELENA)

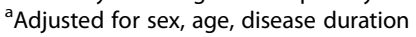


negatively affects every dimension assessed by the SF-36, with the exception of the perceived general health. Disease activity assessed by the physician with a 4-Likert-scale ranging from inactive to very active disease had a similar impact on HRQoL outcomes, with the difference that it was more closely associated with bodily pain and the physical component summary. SELENA-SLEDAI on the other hand was more closely associated with the mental component summary. Overall, global disease activity predominantly affected role physical and role emotional functioning as well as bodily pain. We used the SELENA-SLEDAI organ system classification [27] to differentiate which type of SLE activity impacted most on HRQoL. Active musculoskeletal and renal lupus had a negative influence on most SF-36 dimensions. The impacts of SLE musculoskeletal and renal involvements on HRQoL have been previously reported: In a retrospective study of 303 patients, musculoskeletal flares in the preceding year were independently associated with impairment of most of the subscales of the SF-36, except role limitation due to physical problems and mental health [29]. This is in contrast with our findings, where active musculoskeletal involvement also impaired physical role and mental health, but had no significant effect on physical functioning, general health and vitality. This contrast could be explained by the 4-week window used in our study to assess disease activity, the definition of musculoskeletal involvement based on the SELENA-SLEDAI [30] and by socio-cultural differences in the populations studied. We also found that activity assessed by PGA was more closely correlated with bodily pain than the SELENA-SLEDAI, which emphasizes the importance of the physician's impression in globally assessing SLE patients. In accordance with previous studies our results underline that controlling musculoskeletal activity is of major importance when aiming at improving HRQoL in SLE.

Only few studies have addressed the impact of lupus nephritis on HRQoL. Vu et al. showed in 1999 that patients with lupus nephritis who progress to end stage renal disease have reduced physical functioning and general health subscales, while mental health appear to improve [31]. Strand et al. reported an improvement of HRQoL in SLE patients treated with sodium abetimus. Strikingly, they also found that the role emotional dimension was significantly reduced in lupus nephritis and that after treatment of renal flares this dimension was also the one to improve the most [32]. More recently, Hanly et al. evaluated the consequence of lupus nephritis on HRQoL. Despite no significant difference in HRQoL outcomes between patients with lupus nephritis and those with nonrenal SLE, they showed that patients with advanced renal failure had lower SF-36 subscales (mainly role physical) and summary component scales [33, 34]. Our work shows that among different organs systems assessed for SLE activity, active lupus nephritis had the most pronounced effect on role physical and role emotional functioning. Thus, when assessing response to treatment in patients with lupus nephritis, not only disease activity should be measured but also HRQoL outcomes. Interestingly we found that complement consumption and/or presence of anti-dsDNA antibodies were associated with a decrease in mental health and vitality subscales. The increase in fatigue in immunologically active disease also recalls the results of the BLISS studies, where patients with immunologically active SLE had a better response to belimumab in terms of HRQoL [7]. Altogether these observations may strengthen the impression that immunologic disturbances in SLE are directly responsible for patients' fatigue and mental alteration. One could hypothesize that pathogenic auto-antibodies, and in particular those targeting dsDNA may exert an effect on the central nervous system [35]. We did not find a correlation between active central nervous system involvement and HRQoL, but this analysis was limited by the fact that only few patients had overt neurologic involvement. Hanly et al. also extensively studied the impact of psychiatric and neurological SLE symptoms on HRQoL. They found that mood disorders and headaches were associated with lower mental and physical component summaries, whereas seizures did not impact on HRQoL outcomes. They however found no correlation between the SLEDAI-2 K score or lupus auto-antibodies and HRQoL [11, 36, 37].

Our study has some limitations. Indeed, most patients in our study population had long-standing SLE. Still our results regarding disease activity are similar to those reported in a recent inception cohort study, where patients with active SLE had poorer HRQOL outcomes [5]. Also, while up to $75 \%$ of our patients had been diagnosed with SLE two years or more prior to assessment, HRQoL outcomes in the above mentioned longitudinal study did not change significantly from two years after diagnosis onwards [5]. Thus, we believe that our results regarding disease activity are valid despite the cross-sectional design. We were not able to assess factors such as educational level and presence of fibromyalgia, which are known to negatively impact on HRQoL [18, 38]. Lastly, our study was not able to address the chronicity of SLE, which will need repeated assessment of both disease activity and HRQOL over a longer period.

\section{Conclusions}

In conclusion, our study confirms a low HRQoL in a large cohort of Swiss SLE patients. Disease activity assessed by SELENA-SLEDAI, PGA and ESR all negatively correlate with most HRQoL outcomes. HrQoL was decreased in those with active musculoskeletal and renal involvement and in the presence of classical markers of biological activity. 


\section{Additional file}

Additional file 1: Table S1. Impact of disease activity by organ systems assessed through the SELENA-SLEDAI on health-related quality of life outcomes in 252 patients with systemic lupus erythematosus. (DOCX 96 kb)

\section{Abbreviations}

ACR: American College of Rheumatology; BMI: Body mass index; BP: Bodily pain; GH: General health; MCS: Mental components summaries; $\mathrm{MH}$ : Mental health; PCS: Physical components summaries; PF: Physical performance: PGA: Physician's Global Assessment; QoL: Quality of life; RE: Emotional problems; RP: Physical limitations; SCQM: Swiss Clinical Quality Management Program for Rheumatoid Arthritis; SELENA: Safety of Estrogens in Lupus Erythematosus National Assessment; SF: Social functioning; SF-36: Medical Outcome Study 36-item Short Form; SLE: Systemic lupus erythematosus; SLEDAI: SLE Disease Activity Index; SSCS: Swiss SLE Cohort Study; VT: Vitality

\section{Acknowledgments}

We thank Petra Otto, Nicola Reuschling, Kristin Schmiedeberg, Milos Antic, Heino Prillwitz, Gediminas Matulis, Franziska Mueller, Lea Mazenauer, Marcel Tschopp, Jacqueline Krane-Nuber, Claudio Thurneysen, Christoph Ackermann, Anita Rusch, Christine Sturm, Annette Leimgruber, François Spertini, Stephanie Petitpierre, Guillaume Buss, Denis Comte, Julien Vionnet, Christa-Maria Maniu, Alba Santos, Alice Horisberger, Sylvie Froidevaux, Marie-Astrid Peters, Peter Jandus, Charis Ziori, Marcel Bergmann, Magdalena Posa, Avigael Benhamou, Igor Widdiker, Federico Simonetta, Fanny Fasnacht, David Spoerl, Danielle Allali, Julien Ducommun, Joerg Seebach, Sandra Paiano, Donatella Ciuffreda, Leila Motteli, Axel Finckh, Kiril Zamfirov, Lionel Arlettaz, Sylvie Revaz, Ute Eisenberger, Gregor Lindner, Inge Schudel, Katrin Koenig, Leonore Branco, Andreas Jehle, Andreas Kistler, Martina Pechula, Clemens Cohen for providing clinical data; Emilie Mueller, Isabelle Koenig, Donatienne Wynar, Virginie Ancrenaz, Susete Vieira, Ulla Schuepbach, Zerina Hadzic, Monika Buerki, Anna Christen, Melinda Maeder, Emmanuelle Paccou, Marian Struker, and Silvia Forrer for data collection, Philippe Baumann for data extraction, and all the patients that have contributed to this study.

\section{Funding}

This work was supported by the Gebert-Rüf Foundation (grant number GRS-027/07) and by an unrestricted research grant by Glaxo-Smith-Klein.

\section{Availability of data and materials}

Not applicable.

\section{Authors' contributions}

BC, CC, TP, CR, MT, UHD, ED, TS, JVK, made substantial contributions to conception and design, or acquisition of data, or analysis and interpretation of data. BC, CC, TP, CR, MT, UHD, ED, TS, JVK have been involved in drafting the manuscript or revising it critically for important intellectual content. BC, $C C, T P, C R, M T, U H D, E D, T S, J V K$ have given final approval of the version to be published. Each author have participated sufficiently in the work to take public responsibility for appropriate portions of the content. BC, CC, CR, agreed to be accountable for all aspects of the work in ensuring that questions related to the accuracy or integrity of any part of the work are appropriately investigated and resolved. All authors read and approved the final manuscript.

\section{Competing interests}

The authors declare that they have no competing interests.

\section{Consent for publication}

Not applicable.

\section{Ethics approval and consent to participate}

Written informed consent was obtained from each participant. This study was approved by the ethical committee of the institutions involved and was conducted according to the Declaration of Helsinki. The names of all ethical bodies that approved the study are listed in the Additional file 1: Table S1.

\section{Publisher's Note}

Springer Nature remains neutral with regard to jurisdictional claims in published maps and institutional affiliations.

\section{Author details}

${ }^{1}$ Department of Internal Medicine Specialties, Clinical Immunology and Allergy, University Hospital and School of Medicine, Geneva, Switzerland. ${ }^{2}$ Department of Community Health and Medicine, Clinical Epidemiology, University Hospital and School of Medicine, Geneva, Switzerland. ${ }^{3}$ Division of Internal Medicine and Clinical Immunology Lab, Department of Biomedicine, University Hospital Basel, Basel, Switzerland. ${ }^{4}$ Nephrology, Hypertension and Clinical Pharmacology, University Hospital Bern, Bern, Switzerland. ${ }^{5}$ Clinical Immunology and Allergy, Sion Hospital, Sion, Switzerland. 'Rheumatology, Kantonsspital Schaffhausen, Schaffhausen, Switzerland. ${ }^{7}$ Division of Rheumatology and Immunology, Department of Internal Medicine, Kantonsspital St Gallen, St Gallen, Switzerland. ${ }^{8}$ Clinical Immunology and Allergy, University Hospital Lausanne, Rue du Bugnon 46, Lausanne 1011, Switzerland

Received: 24 October 2016 Accepted: 16 March 2017

Published online: 28 March 2017

\section{References}

1. Lisnevskaia L, Murphy G, Isenberg D. Systemic lupus erythematosus. Lancet. 2014;384(9957):1878-88

2. Ribi C, Trendelenburg M, Gayet-Ageron A, Cohen C, Dayer E, Eisenberger U, et al. The Swiss Systemic lupus erythematosus Cohort Study (SSCS) - crosssectional analysis of clinical characteristics and treatments across different medical disciplines in Switzerland. Swiss Med Wkly. 2014;144:w13990.

3. Katz P, Morris A, Trupin L, Yazdany J, Yelin E. Disability in valued life activities among individuals with systemic lupus erythematosus. Arthritis Rheum. 2008;59(4):465-73

4. Kozora E, Ellison MC, West S. Depression, fatigue, and pain in systemic lupus erythematosus (SLE): relationship to the American College of Rheumatology SLE neuropsychological battery. Arthritis Rheum. 2006:55(4):628-35.

5. Urowitz M, Gladman DD, Ibañez D, Sanchez-Guerrero J, Bae SC, Gordon C, et al. Changes in quality of life in the first 5 years of disease in a multicenter cohort of patients with systemic lupus erythematosus. Arthritis Care Res (Hoboken). 2014;66(9):1374-9.

6. Jolly M. How does quality of life of patients with systemic lupus erythematosus compare with that of other common chronic illnesses? I Rheumatol. 2005:32(9):1706-8.

7. Strand V, Levy RA, Cervera R, Petri MA, Birch H, Freimuth WW, et al. Improvements in health-related quality of life with belimumab, a Blymphocyte stimulator-specific inhibitor, in patients with autoantibodypositive systemic lupus erythematosus from the randomised controlled BLISS trials. Ann Rheum Dis. 2014;73(5):838-44.

8. Touma Z, Gladman DD, Ibañez D, Urowitz MB. Is there an advantage over SF-36 with a quality of life measure that is specific to systemic lupus erythematosus? J Rheumatol. 2011;38(9):1898-905.

9. Devilliers H, Amoura Z, Besancenot J-F, Bonnotte B, Pasquali J-L, Wahl D, et al. Responsiveness of the 36-item Short Form Health Survey and the Lupus Quality of Life questionnaire in SLE. Rheumatology (Oxford). 2015; 54(5):940-9

10. Strand V, Petri M, Kalunian K, Gordon C, Wallace DJ, Hobbs K, et al. Epratuzumab for patients with moderate to severe flaring SLE: healthrelated quality of life outcomes and corticosteroid use in the randomized controlled ALLEVIATE trials and extension study SL0006. Rheumatology (Oxford). 2014:53(3):502-11.

11. Hanly JG, Urowitz MB, O'Keeffe AG, Gordon C, Bae S-C, Sanchez-Guerrero J, et al. Headache in systemic lupus erythematosus: results from a prospective, international inception cohort study. Arthritis Rheum. 2013;65(11):2887-97.

12. Freire E, Bruscato A, Ciconelli R. Quality of life in systemic lupus erythematosus patients in Northeastern Brazil: Is health-related quality of life a predictor of survival for these patients? Acta Reumatol Port 2009:34(2A):207-11.

13. Hanly JG. Disease activity, cumulative damage and quality of life in systematic lupus erythematosus: results of a cross-sectional study. Lupus. 1997;6(3):243-7.

14. Gladman DD, Urowitz MB, Ong A, Gough J, Mackinnon A. Lack of correlation among the 3 outcomes describing SLE: disease activity, damage and quality of life. Clin Exp Rheumatol. 1996;14(3):305-8. 
15. Stoll T, Gordon C, Seifert B, Richardson K, Malik J, Bacon PA, et al. Consistency and validity of patient administered assessment of quality of life by the MOS SF-36; its association with disease activity and damage in patients with systemic lupus erythematosus. J Rheumatol. 1997;24(8):1608-14.

16. Thumboo J, Fong KY, Ng TP, Leong KH, Feng PH, Thio ST, et al. Validation of the MOS SF-36 for quality of life assessment of patients with systemic lupus erythematosus in Singapore. J Rheumatol. 1999;26(1):97-102.

17. Alarcón GS, Mcgwin G, Uribe A, Friedman AW, Roseman JM, Fessler BJ, et al. Systemic lupus erythematosus in a multiethnic lupus cohort (LUMINA). XVII. Predictors of self-reported health-related quality of life early in the disease course. Arthritis Rheum. 2004;51(3):465-74.

18. Fortin PR, Abrahamowicz M, Neville C, du Berger R, Fraenkel L, Clarke AE, et al. Impact of disease activity and cumulative damage on the health of lupus patients. Lupus. 1998;7(2):101-7.

19. Gilboe IM, Kvien TK, Husby G. Disease course in systemic lupus erythematosus: changes in health status, disease activity, and organ damage after 2 years. J Rheumatol. 2001;28(2):266-74.

20. Chizzolini C, Cohen CD, Eisenberger U, Hauser T, Hunziker T, Leimgruber A, et al. Towards the Swiss systemic lupus erythematosus cohort study (SSCS). Rev Med Suisse. 2009;5(199):808-11.

21. Hochberg MC. Updating the American College of Rheumatology revised criteria for the classification of systemic lupus erythematosus. Arthritis Rheum. 1997;40(9):1725.

22. Tan EM, Cohen AS, Fries JF, Masi AT, Mcshane DJ, Rothfield NF, et al. The 1982 revised criteria for the classification of systemic lupus erythematosus. Arthritis Rheum. 1982;25(11):1271-7.

23. Petri M, Orbai A-M, Alarcón GS, Gordon C, Merrill JT, Fortin PR, et al. Derivation and validation of the Systemic Lupus International Collaborating Clinics classification criteria for systemic lupus erythematosus. Arthritis Rheum. 2012;64(8):2677-86.

24. Ware JE, The SCD, MOS. 36-item short-form health survey (SF-36). I. Conceptual framework and item selection. Med Care. 1992;30(6):473-83.

25. Mchorney CA, Ware JE, Lu JF, The SCD, MOS. 36-item Short-Form Health Survey (SF-36): III. Tests of data quality, scaling assumptions, and reliability across diverse patient groups. Med Care. 1994;32(1):40-66.

26. Richard J-L, Bouzourène $K$, Gallant $S$, Ricciardi $P$, Sudre $P$, Iten $A$, et al. Validation et normes du SF-36 dans la population du canton de Vaud. Raisons de santé. 2000;28(1):1:61.

27. Petri M, Kim MY, Kalunian KC, Grossman J, Hahn BH, Sammaritano LR, et al. Combined oral contraceptives in women with systemic lupus erythematosus. N Engl J Med. 2005;353(24):2550-8.

28. Touma Z, Urowitz MB, Ibañez D, Gladman DD. SLEDAI-2 K 10 days versus SLEDAI-2 K 30 days in a longitudinal evaluation. Lupus. 2011;20(1):67-70

29. Zhu TY, Tam L-S, Lee WWY, Lee KK, Li EK. Relationship between flare and health-related quality of life in patients with systemic lupus erythematosus. J Rheumatol. 2010;37(3):568-73.

30. Bombardier C, Gladman DD, Urowitz MB, Caron D, Chang CH. Derivation of the SLEDAl. A disease activity index for lupus patients. The Committee on Prognosis Studies in SLE. Arthritis Rheum. 1992:35(6):630-40.

31. Vu TV, Escalante A. A comparison of the quality of life of patients with systemic lupus erythematosus with and without endstage renal disease. J Rheumatol. 1999;26(12):2595-601.

32. Strand V, Aranow C, Cardiel MH, Alarcón-Segovia D, Furie R, Sherrer Y, et al. Improvement in health-related quality of life in systemic lupus erythematosus patients enrolled in a randomized clinical trial comparing LP 394 treatment with placebo. Lupus. 2003;12(9):677.

33. Hanly JG, O'Keeffe AG, Su L, Urowitz MB, Romero-Diaz J, Gordon C, et al. The frequency and outcome of lupus nephritis: results from an international inception cohort study. Rheumatology. 2016;55(2):252-62.

34. Mak A, Tay SH. Outcome of lupus glomerulonephritis: the role of prospective observational cohort studies. Rheumatology (Oxford). 2016:55(2):195-6.

35. Colasanti T, Delunardo F, Margutti P, Vacirca D, Piro E, Siracusano A, et al. Autoantibodies involved in neuropsychiatric manifestations associated with systemic lupus erythematosus. J Neuroimmunol. 2009:212(1-2):3-9.
36. Hanly JG, Fisk JD, McCurdy G, Fougere L, Douglas J-A. Neuropsychiatric syndromes in patients with systemic lupus erythematosus and rheumatoid arthritis. J Rheumatol. 2005;32(8):1459-6.

37. Hanly JG, Su L, Urowitz MB, Romero-Diaz J, Gordon C, Bae S-C, et al. Mood Disorders in Systemic Lupus Erythematosus: Results From an International Inception Cohort Study. Arthritis Rheumatol. 2015;67(7):1837-47.

38. Gladman DD, Urowitz MB, Gough J, Mackinnon A. Fibromyalgia is a major contributor to quality of life in lupus. J Rheumatol. 1997;24(11):2145-8.

\section{Submit your next manuscript to BioMed Central and we will help you at every step:}

- We accept pre-submission inquiries

- Our selector tool helps you to find the most relevant journal

- We provide round the clock customer support

- Convenient online submission

- Thorough peer review

- Inclusion in PubMed and all major indexing services

- Maximum visibility for your research

Submit your manuscript at www.biomedcentral.com/submit
Biomed Central 